\title{
Digital sum moments and substitutions
}

\author{
by \\ Jean Marie Dumont and Alain Thomas (Marseille)
}

0. Introduction. In an article published in 1986 Jean Coquet ([C86]) proved that if $s_{2}(\nu)$ denotes the sum of binary digits of $\nu$, then for each integer $k$ there exist 1-periodic bounded functions $F_{k, 0}, F_{k, 1}, \ldots, F_{k, k-1}$ such that for any integer $x$ one has, with $l=\log _{2} x$,

$$
x^{-1} \sum_{\nu<x}\left(s_{2}(\nu)\right)^{k}=(l / 2)^{k}+\sum_{h<k} l^{h} F_{k, h}(l) .
$$

The result in the case $k=1$ was first established by H. Delange ([De75]) and has recently been generalized to the case of " $\theta$-expansion of positive integers" with $\theta$ an arbitrary real base $>1$ ([GTi91]), with an $o(1)$ correction term; in this case the function $F_{k, 0}$ is shown to be continuous; so are $F_{k, 0}$ and $F_{k, 1}$ in the case $k=2$, for the binary expansion (see [C86] and [K90]).

Our first aim in this paper is to give a generalization of (1), including the proof of the continuity of the functions $F_{k, h}$, within the framework of the so-called "numeration system associated with a substitution".

More precisely, if $\sigma$ is a primitive substitution on a finite alphabet $\mathcal{A}$ whose largest eigenvalue satisfies $\theta>1$, and $s^{f}(\nu)$ denotes $\sum_{i=1}^{n} f\left(m_{i}\right)$ (where for $\nu$ an integer, $\sum_{i=1}^{n}\left|\sigma^{i-1}\left(m_{i}\right)\right|$ is the unique admissible representation of $\nu$, and $f$ is a map from $\mathcal{A}^{*}$ to $\mathbb{R}$ ), then we prove the existence of a real number $\alpha$ and 1-periodic continuous functions $F_{k, h}(h=0,1, \ldots, k-1)$ such that for any $x>0$,

$$
x^{-1} \sum_{\nu<x}\left(s^{f}(\nu)\right)^{k}=\alpha^{k} l^{k}+\sum_{h<k} l^{h} F_{k, h}(l)+\varepsilon(x)
$$

where $\lim _{x \rightarrow \infty} \varepsilon(x)=0$, and $l=\log _{\theta} x$.

Moreover, we prove a similar formula for "moments of the sum-of-digits function" in the form

(3) $x^{-1} \sum_{\nu<x}\left(s^{f}(\nu)-\alpha l\right)^{2 k}=(2 k-1)(2 k-3) \ldots 1 \beta^{k} l^{k}+\sum_{h<k} l^{h} G_{k, h}(l)+\eta(x)$

where the real number $\beta$ is explicitly determined and $\lim _{x \rightarrow+\infty} \eta(x)=0$, the 
functions $G_{k, h}$ being 1-periodic and continuous. For odd moments $(2 k-1$ in place of $2 k$ on the l.h.s. of (3)) the first term of the r.h.s. of (3) disappears.

The functions $F_{k, h}$ of (2) are shown to be nowhere differentiable when $\alpha \neq 0$ and $\alpha \neq f(\omega)$ ( $\omega$ being the empty word); the functions $G_{k, h}$ of (3) are also nowhere differentiable if $\beta \neq 0$ and $\alpha \neq f(\omega)$.

Note that the constants $\alpha$ and $\beta$ (in the case $\alpha=0$ ) of the formulae (2) and (3) were previously determined (in [D90]) by another method; moreover, the special form of the moments of the sum-of-digits function is clearly connected with the gaussian distribution of this function, and was first conjectured by J. M. Luck ([L]); we hope to give a more precise statement of this idea in a forthcoming paper.

The general framework of "substitutions on a finite alphabet" allows us to study some mathematical models useful in theoretical physics; in some of these cases we give the explicit values of the constants $\alpha$ and $\beta$.

To return to the initial formula (1), we also note that when $\mathcal{A}=\{1\}$ and $\sigma(1)=11 \ldots 1$ ( $q$ times one, $q$ an integer $\geq 2$ ), the substitutive numeration coincides with the ordinary base $q$ numeration, and in this case the remainder terms $\varepsilon(x)$ and $\eta(x)$ of (2) and (3) disappear when $x$ is an integer, according to (1); moreover, we then have $\alpha=(q-1) / 2$ and $\beta=\left(q^{2}-1\right) / 12$ (if $q=2$, the result for $\beta$ agrees with that of P. Kirschenhofer ([K90]).

With the substitutive numeration system, one can also obtain expansions of integers with respect to linear recurrences studied in [GTi91] (see also [Sh88] and [B89]); we make explicit that connection, and give the values of $\alpha$ and $\beta$ in the last section. For instance, in the Fibonacci case $\alpha=(5-\sqrt{5}) / 10$ (in accordance with [CV86]) and $\beta=1 /(5 \sqrt{5})$.

Finally, we note that in the case of ordinary $q$-adic expansion ( $q$ an integer $\geq 2$ ) some asymptotic formulae involving the sum of digits can also be obtained by other methods, issuing from analytic theory of numbers (cf. $[\mathrm{FG}]$ and $[\mathrm{MM}])$.

\section{Numeration system and sums associated with a substitution.} Let $\sigma$ be a substitution on a finite alphabet $\mathcal{A}=\{1,2, \ldots, d\}$, i.e. a map from $\mathcal{A}$ to $\mathcal{A}^{*} \backslash \omega$, the set of non-empty words of $\mathcal{A}$; let $M$ be the transpose of its matrix $\left(M_{a, b}\right.$ is the number of occurrences of $b$ in $\left.\sigma a\right) ;|m|$ denotes the length of $m \in \mathcal{A}^{*}$. We assume that $M$ is primitive and that the word $\sigma(1)$ has length at least 2 and begins with the letter 1 . By the theorem of Perron-Frobenius, there exists a unique eigenvalue $\theta$ of $M$ with maximum modulus. As $|\sigma(1)| \geq 2$, one has $\theta>1$.

We first recall a representation of integers ([DT89]) which we reset in terms of automata. The state set of the prefix automaton is the alphabet $\mathcal{A}$ of the substitution. The alphabet of the automaton is the set $\mathcal{A}^{\prime}$ of words $m$ such that $\exists a \in \mathcal{A}, m<\sigma a$. There is one arc from $a$ to $b$ with label $m$ iff 
$m b \leq \sigma a$. We write $b=a \cdot m$. Every sequence $\left(m_{n}, \ldots, m_{1}\right), m_{i} \in \mathcal{A}^{\prime}$, which is the label of a path with initial state $a$ is called a-recognizable. There are exactly $\left|\sigma^{n}(a)\right| a$-recognizable sequences of length $n$.

Since $1<\sigma(1)$, for each integer $\nu \geq 1$ there exists a unique 1-recognizable sequence $\left(m_{n}, \ldots, m_{1}\right)$ such that $\nu=\sum_{i=1}^{n}\left|\sigma^{i-1}\left(m_{i}\right)\right|$ and $m_{n}$ is not empty; this sequence is called the (admissible) representation of $\nu$.

For instance, a special case is the representation in the Fibonacci base $\left(F_{0}=1, F_{1}=2, F_{i+1}=F_{i}+F_{i-1}\right)$. Indeed, if $\sigma$ is defined by $\sigma(1)=12$ and $\sigma(2)=1$, then the $m_{i}$ are $\omega$ (empty word) or 1 , and $\left|\sigma^{i-1}\left(m_{i}\right)\right|$ is 0 if $m_{i}=\omega$, and $F_{i-1}$ if $m_{i}=1$; the prefix automaton recognizes the sequences $\left(m_{n}, \ldots, m_{1}\right)$ such that $m_{i+1} m_{i} \neq 11$ (see Fig. 1).

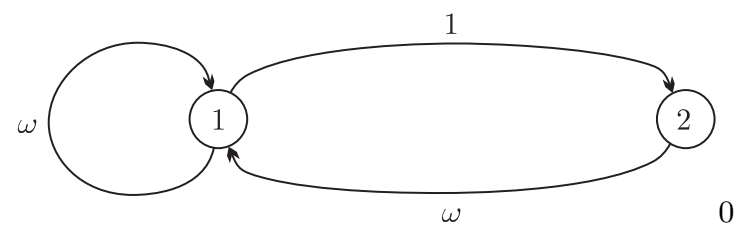

Fig. 1

The $q$-ary representation ( $q$ an integer, $q \geq 2$ ) is another special case, with the substitution defined on $\mathcal{A}=\{1\}$ by $\sigma(1)=1^{q}$ (see Fig. 2).

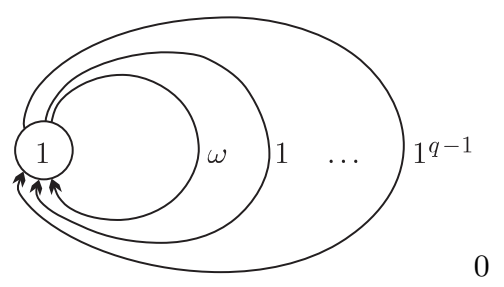

Fig. 2

We define the sum-of-digits function relative to a map $f: \mathcal{A}^{*} \rightarrow \mathbb{R}$ by

$$
s^{f}(\nu)=\sum_{i=1}^{n} f\left(m_{i}\right) \quad\left(\left(m_{n}, \ldots, m_{1}\right) \text { being the representation of } \nu\right) \text {. }
$$

A moment of order $k$ is

$$
S_{k, \lambda}^{f}(x)=\sum_{0<\nu<x}\left(s^{f}(\nu)-\lambda \log _{\theta} x\right)^{k} \quad \text { for } x \in \mathbb{R}_{+}^{*} \text { and } k \in \mathbb{N}, \lambda \in \mathbb{R} .
$$

We will also use, for the computation of the asymptotic expansion of $S_{k, \lambda}^{f}(x)$, the vector $V_{n}^{k}$ defined by

$$
\left(V_{n}^{k}\right)_{a}=\sum_{m_{n}, \ldots, m_{1}}\left(g\left(m_{n}\right)+\ldots+g\left(m_{1}\right)\right)^{k} \quad \text { for any } a \in \mathcal{A}, n \text { an integer } \geq 1
$$


( $g$ defined by $g(m)=f(m)-\lambda$; summation over all $a$-recognizable sequences of length $n$ ),

$$
\left(V_{0}^{k}\right)_{a}= \begin{cases}0 & \text { if } k \neq 0 \\ 1 & \text { if } k=0\end{cases}
$$

and the matrix $A^{(k)}$ defined by

$$
A_{a, b}^{(k)}=\sum_{m} g(m)^{k} \quad\left(\text { sum over all } m \in \mathcal{A}^{*} \text { such that } m b \leq \sigma a\right) .
$$

When $f$ is a morphism and $f \circ \sigma=f$ (this means $f\left(m m^{\prime}\right)=f(m)+f\left(m^{\prime}\right)$ and $f(\sigma(m))=f(m)$ for all $m, m^{\prime}$ in $\left.\mathcal{A}^{*}\right)$, one has

$$
S_{k, \lambda}^{f}(x)=\sum_{0<\nu<x}\left(f\left(u_{1} \ldots u_{\nu}\right)-\lambda \log _{\theta} x\right)^{k},
$$

$\left(u_{n}\right)_{n \geq 1}$ being the fixed point of $\sigma$ extended by concatenation to $\mathcal{A}^{\mathbb{N}}$, with $u_{1}=1$. Indeed (see [DT89]), if $u_{1} \ldots u_{\nu}=\sigma^{n-1}\left(m_{n}\right) \ldots \sigma^{0}\left(m_{1}\right)$, then $f\left(u_{1} \ldots u_{\nu}\right)=s^{f}(\nu)$. We note that a formula for $\sum f\left(u_{1} \ldots u_{\nu}\right)$, with weaker assumptions on $f$, was given in [DT91].

\section{Computation of $V_{n}^{k}$}

LEMMA 1.

$$
V_{n+1}^{k}=M V_{n}^{k}+\sum_{h<k}\left(\begin{array}{l}
k \\
h
\end{array}\right) A^{(k-h)} V_{n}^{h} .
$$

Proof. From the definition of $V_{n}^{k}$ we deduce

$$
\left(V_{n+1}^{k}\right)_{a}=\sum_{m_{n+1}, b} \sum_{m_{n}, \ldots, m_{1}}\left(g\left(m_{n+1}\right)+\ldots+g\left(m_{1}\right)\right)^{k}
$$

(sum over $\left(m_{n+1}, b\right)$ such that $m_{n+1} b \leq \sigma a$, and over $m_{n}, \ldots, m_{1} b$-recognizable). By the binomial formula,

$$
\left(g\left(m_{n+1}\right)+\ldots+g\left(m_{1}\right)\right)^{k}=\sum_{h \leq k}\left(\begin{array}{l}
k \\
h
\end{array}\right)\left(g\left(m_{n+1}\right)\right)^{k-h}\left(g\left(m_{n}\right)+\ldots+g\left(m_{1}\right)\right)^{h} .
$$

Thus

$$
V_{n+1}^{k}=\sum_{h \leq k}\left(\begin{array}{l}
k \\
h
\end{array}\right) A^{(k-h)} V_{n}^{h} .
$$

As $A^{(0)}=M$, we obtain the assertion.

Remark 1 . If $k=0$, this relation becomes $V_{n+1}^{0}=M V_{n}^{0}$; hence

$$
V_{n}^{0}=M^{n}\left(\begin{array}{c}
1 \\
\vdots \\
1
\end{array}\right)=\left(\left|\sigma^{n}(a)\right|\right)_{a \in \mathcal{A}} .
$$


Let

$$
P(X)=\prod_{j=1}^{d^{\prime}}\left(X-\theta_{j}\right)^{\alpha_{j}}
$$

be the minimal polynomial of the matrix $M$, with $\theta=\theta_{1}>\left|\theta_{2}\right| \geq\left|\theta_{3}\right|$ $\geq \ldots \geq\left|\theta_{d^{\prime}}\right|$ and $\theta_{j} \neq \theta_{j^{\prime}}$ for $j \neq j^{\prime}$. The following lemma states that the sequence $V^{k}=\left(V_{n}^{k}\right)_{n \in \mathbb{N}}$ satisfies a recurrence equation related to the polynomial $P(X)^{k+1}$.

Let $S:\left(\mathbb{C}^{d}\right)^{\mathbb{N}} \rightarrow\left(\mathbb{C}^{d}\right)^{\mathbb{N}}$ be the shift (defined by $S\left(\left(x_{n}\right)_{n \in \mathbb{N}}\right)=$ $\left.\left(x_{n+1}\right)_{n \in \mathbb{N}}\right)$.

Lemma 2. The sequence $V^{k}=\left(V_{n}^{k}\right)_{n \in \mathbb{N}}$ satisfies $(P(S))^{k+1}\left(V^{k}\right)=0$.

Proof. This is true for $k=0$ : by Remark $1, V^{0}$ is the sequence $n \rightarrow$ $M^{n}\left(\begin{array}{c}1 \\ \vdots \\ 1\end{array}\right)$, hence $P(S)\left(V^{0}\right)$ is the sequence $n \rightarrow(P(M)) M^{n}\left(\begin{array}{c}1 \\ \vdots \\ 1\end{array}\right)$; now $P(M)=0$. Suppose the lemma to be true for any $h \leq k-1$. The formula of Lemma 1 can be written as

$$
S\left(V^{k}\right)=M V^{k}+\sum_{h<k}\left(\begin{array}{l}
k \\
h
\end{array}\right) A^{(k-h)} V^{h} .
$$

Applying $(P(S))^{k}$ and using the fact that $(P(S))^{k}$ commutes with any matrix, we obtain

$$
\left((P(S))^{k} \circ S\right)\left(V^{k}\right)=(P(S))^{k}\left(M V^{k}\right) .
$$

But $(P(S))^{k} \circ S=S \circ(P(S))^{k}$ and $(P(S))^{k}\left(M V^{k}\right)=M\left((P(S))^{k}\left(V^{k}\right)\right)$, so we have

$$
S\left(W^{k}\right)=M W^{k}, \quad \text { where } W^{k}=(P(S))^{k}\left(V^{k}\right) .
$$

We deduce by induction $S^{i}\left(W^{k}\right)=M^{i} W^{k}$ for all $i \in \mathbb{N}$. Hence

$$
(P(S))\left(W^{k}\right)=(P(M)) W^{k} \text {, i.e. }(P(S))^{k+1}\left(V^{k}\right)=0 W^{k}=0 .
$$

Lemma 3. There exist polynomials $p_{j k a}(X) \in \mathbb{C}[X]$ such that

(i) $\left(V_{n}^{k}\right)_{a}=\sum_{j=1}^{d^{\prime}} p_{j k a}(n) \theta_{j}^{n}$ for any $a \in \mathcal{A}$,

(ii) $\left(V_{n}^{k}\right)_{a}=p_{1 k a}(n) \theta^{n}+O\left(n^{k^{\prime}}\left|\theta_{2}\right|^{n}\right)$ with $k^{\prime}=(k+1) \alpha_{2}-1$,

(iii) $p_{1 k a}(X) \in \mathbb{R}[X]$ and $d^{\circ}\left(p_{1 k a}\right) \leq k$.

Proof. (i) Let $V^{k, a}$ be the sequence $n \rightarrow\left(V_{n}^{k}\right)_{a}$. Lemma 2 implies $\left(P\left(S^{\prime}\right)\right)^{k+1}\left(V^{k, a}\right)=0$, where $S^{\prime}$ is the shift on $\mathbb{C}^{\mathbb{N}}$. It is known that the kernel of $\left(P\left(S^{\prime}\right)\right)^{k+1}$ is generated by the sequences $n \rightarrow n^{l} \theta_{j}^{n}$, with $1 \leq j \leq d^{\prime}$ and $l$ less than the order of $\theta_{j}$ in $(P(X))^{k+1}$, i.e. $l<(k+1) \alpha_{j}$. In other words, there exist polynomials $p_{j k a}(X)$ of degree at most $(k+1) \alpha_{j}-1$, satisfying (i). 
(iii) We have

$$
\left(V_{n}^{k}\right)_{a}=\overline{\left(V_{n}^{k}\right)_{a}}=\sum_{j=1}^{d^{\prime}} \overline{p_{j k a}(n)} \overline{\theta_{j}^{n}} \quad \text { and } \overline{\theta_{1}}=\theta_{1} .
$$

Using the unicity of the decomposition of $V^{k, a}$, we obtain $\overline{p_{1 k a}(n)}=p_{1 k a}(n)$ for all $n \in \mathbb{N}$, so $p_{1 k a}(X) \in \mathbb{R}[X]$. We have $\alpha_{1}=1$ by Perron-Frobenius, hence $d^{\circ}\left(p_{1 k a}\right) \leq(k+1)-1$.

Remark 2 . For $k=0$, the polynomial $p_{10 a}(X)$ has degree 0 , i.e. it is a constant $\varepsilon_{a}$. The relation (ii) becomes

$$
\left(V_{n}^{0}\right)_{a}=\varepsilon_{a} \theta^{n}+O\left(n^{\alpha_{2}-1}\left|\theta_{2}\right|^{n}\right) .
$$

Using Remark 1 we deduce

$$
\varepsilon_{a}=\lim _{n \rightarrow+\infty} \theta^{-n}\left|\sigma^{n}(a)\right| .
$$

For every word $m=a_{1} \ldots a_{n}$, we will denote by $\varepsilon(m)$ the $\operatorname{sum} \sum_{i=1}^{n} \varepsilon_{a_{i}}$.

\section{Asymptotic expansion for $S_{k, \lambda}^{f}(x)$}

Proposition 1. There exist bounded functions $F_{k, h}: \mathbb{R} \rightarrow \mathbb{R}$ with period 1 such that

$$
\begin{gathered}
S_{k, \lambda}^{f}(x)=x \sum_{h=0}^{k} l^{h} F_{k, h}(l)+O\left(\varphi_{k}(l)\right) \quad\left(\text { as } x \rightarrow+\infty, \text { with } l=\log _{\theta} x\right), \\
\varphi_{k}(l)= \begin{cases}l^{k^{\prime}}\left|\theta_{2}\right|^{l} & \text { if }\left|\theta_{2}\right|>1, \text { with } k^{\prime}=(k+1) \alpha_{2}-1, \\
l^{k^{\prime}+1} & \text { if }\left|\theta_{2}\right|=1, \\
l^{k} & \text { if }\left|\theta_{2}\right|<1 .\end{cases}
\end{gathered}
$$

Note that in all cases $\varphi_{k}(l)$ is $o(x)$. The functions $F_{k, k}$ are in fact constants (cf. §5).

We first need a lemma about the representation, relative to a substitution $\sigma$, of real positive numbers.

Lemma 4. For any $x \in \mathbb{R}_{+}^{*}$, there exists an integer $n=n(x)$ and a unique infinite 1-recognizable sequence $\left(m_{n}, m_{n-1}, \ldots\right)$ such that

$$
x=\sum_{i=-\infty}^{n} \varepsilon\left(m_{i}\right) \theta^{i-1},
$$

$m_{n}$ is not empty and $m_{i} a_{i} \neq \sigma\left(a_{i+1}\right)$ for infinitely many $i$ (with $a_{i}=$ $\left.1 \cdot m_{n} \cdot \ldots \cdot m_{i}\right)$. We also have $n(x)=\log _{\theta} x+O(1)$.

P r o of. In [DT89], we define the representation of real numbers belonging to $\left[0, \varepsilon(1)\left[\right.\right.$. Now, if $x \in \mathbb{R}_{+}^{*}$, define $n(x)$ as the unique integer $n$ such that $\varepsilon(1) \theta^{n-1} \leq x<\varepsilon(1) \theta^{n}$. So $x \theta^{-n} \in[0, \varepsilon(1)$ [ and has the representation 
$x \theta^{-n}=\sum_{i=1}^{+\infty} \varepsilon\left(\mu_{i}\right) \theta^{-i} . \mu_{1}$ is not empty, else $x$ would be less than $\varepsilon(1) \theta^{n-1}$. Lemma 4 is thus proved with $m_{i}=\mu_{n-i+1} \forall i \leq n$.

Proof of Proposition 1. We will define $F_{k, h}$ as a sum of two functions $F_{k, h}^{1}$ and $F_{k, h}^{2}$.

In order to define these, we denote by $b_{p}(k, a)$ the coefficients of the polynomial $p_{1 k a}(X)$ of Lemma 3 , i.e.

$$
p_{1 k a}(X)=\sum_{p=0}^{k} b_{p}(k, a) X^{p} .
$$

Given a real number $l, x=\theta^{l}$ has, by Lemma 4 , the representation

$$
x=\sum_{i=-\infty}^{n} \varepsilon\left(m_{i}\right) \theta^{i-1}
$$

and we set $x_{i}=f\left(m_{n}\right)+\ldots+f\left(m_{i+2}\right)-\lambda(l-i)$ for $-\infty<i \leq n-2$. We define

$$
F_{k, h}^{1}(l)=\theta^{-l} \sum_{i, p, q}\left(\begin{array}{l}
k \\
q
\end{array}\right) S(x, i, p, q)\left(\begin{array}{l}
p \\
h
\end{array}\right)(i-l)^{p-h} \theta^{i}
$$

(sum over $-\infty<i \leq n-2$ and $h \leq p \leq q \leq k$ ) with

$$
S(x, i, p, q)=\sum_{m, a}\left(x_{i}+f(m)\right)^{k-q} b_{p}(q, a)
$$

(sum over $(m, a) \in \mathcal{A}^{*} \times \mathcal{A}$ such that $\left.m a \leq m_{i+1}\right)$.

We define $F_{k, h}^{2}$ just as $F_{k, h}^{1}$, with the condition $-\infty<i \leq n-2$ replaced by $-\infty<i \leq n-1$, and with $S(x, i, p, q)$ replaced by

$$
S^{\prime}(x, i, p, q)=\sum_{m, a}(f(m)-\lambda(l-i))^{k-q} b_{p}(q, a)
$$

(sum over $1 \leq m<m a \leq \sigma(1)$ if $i \leq n-2$; over $1 \leq m<m a \leq m_{n}$ if $i=n-1)$. In these definitions, we assume that $0^{0}=1$.

Then we estimate the sum

$$
x \sum_{h=0}^{k} l^{h} F_{k, h}^{1}(l) .
$$

We replace $F_{k, h}^{1}(l)$ by its value and we sum first over $h, 0 \leq h \leq p$, then over $p, 0 \leq p \leq q$. We find that (2) is the sum of

$$
\varphi(i, q, m, a)=\left(\begin{array}{l}
k \\
q
\end{array}\right)\left(x_{i}+f(m)\right)^{k-q} p_{1 q a}(i) \theta^{i}
$$

over $-\infty<i \leq n-2,0 \leq q \leq k$ and $m a \leq m_{i+1}$. 
When $i \geq 0$ and (for example) $l \geq 1$, we use Lemma 3(ii) and the estimate $n=l+O(1)$ of Lemma 4 to obtain

$$
\left|\varphi(i, q, m, a)-\left(\begin{array}{l}
k \\
q
\end{array}\right)\left(x_{i}+f(m)\right)^{k-q}\left(V_{i}^{q}\right)_{a}\right| \leq C l^{k-q}(i+1)^{q^{\prime}}\left|\theta_{2}\right|^{i}
$$

with $C$ a constant independent of $i$ and $l$, and $q^{\prime}=\alpha_{2}(q+1)-1$. When $i \leq-1$ and $l \geq 1$, we have

$$
\left|x_{i}+f(m)\right| \leq C^{\prime}|i| l \quad \text { and } \quad|\varphi(i, q, m, a)| \leq C^{\prime \prime} l^{k-q}|i|^{k} \theta^{i}
$$

with $C^{\prime}$ and $C^{\prime \prime}$ constants. But $\sum_{i=0}^{n-2} l^{k-q}(i+1)^{q^{\prime}}\left|\theta_{2}\right|^{i}$ and $\sum_{i=-\infty}^{-1} l^{k-q}|i|^{k} \theta^{i}$ are $O\left(\varphi_{k}(l)\right)$ as $l$ tends to $+\infty$. Finally, (2) is equal to

$$
\sum_{i, q, m, a}\left(\begin{array}{l}
k \\
q
\end{array}\right)\left(x_{i}+f(m)\right)^{k-q}\left(V_{i}^{q}\right)_{a}+O\left(\varphi_{k}(l)\right)
$$

(sum over $0 \leq i \leq n-2,0 \leq q \leq k$ and $m a \leq m_{i+1}$ ).

Using the definition of $\left(V_{i}^{q}\right)_{a}$ and the binomial formula, it is also equal to

$$
\sum_{i, m, m_{i}^{\prime}, \ldots, m_{1}^{\prime}}\left(x_{i}+f(m)+g\left(m_{i}^{\prime}\right)+\ldots+g\left(m_{1}^{\prime}\right)\right)^{k}+O\left(\varphi_{k}(l)\right)
$$

(sum over $0 \leq i \leq n-2$ and $m_{n} \ldots m_{i+2} m m_{i}^{\prime} \ldots m_{1}^{\prime}$ 1-recognizable, with $\left.m<m_{i+1}\right)$.

These 1-recognizable sequences may be interpreted as the representations of all the integers $\nu$ belonging to the interval $\left\{N_{1}, N_{1}+1, \ldots, N-1\right\}$ (see Section 1), with

$$
N_{1}=\left|\sigma^{n-1}\left(m_{n}\right)\right| \quad \text { and } \quad N=\sum_{i=1}^{n}\left|\sigma^{i-1}\left(m_{i}\right)\right| ;
$$

and (3) as the sum

$$
\sum_{N_{1} \leq \nu<N}\left(s^{f}(\nu)-\lambda l\right)^{k}+O\left(\varphi_{k}(l)\right) .
$$

Now the representations of the integers $\nu$ belonging to $\left\{1,2, \ldots, N_{1}-1\right\}$ are the 1-recognizable sequences $m m_{i}^{\prime} \ldots m_{1}^{\prime}$ such that $0 \leq i \leq n-2$ and $1 \leq m<\sigma(1)$, or $i=n-1$ and $1 \leq m<m_{n}$. We obtain

$$
x \sum_{h \leq k} l^{h} F_{k, h}^{2}(l)=\sum_{0<\nu<N_{1}}\left(s^{f}(\nu)-\lambda l\right)^{k}+O\left(\varphi_{k}(l)\right) .
$$

There remains to estimate $\sum_{N \leq \nu<x}\left(s^{f}(\nu)-\lambda l\right)^{k}$ (or $\sum_{x \leq \nu<N}\left(s^{f}(\nu)-\right.$ $\lambda l)^{k}$ if $\left.x<N\right)$. This sum is $O\left(l^{k}|N-x|\right)$. We have

$$
N-x=\sum_{i=1}^{n}\left(\left|\sigma^{i-1}\left(m_{i}\right)\right|-\varepsilon\left(m_{i}\right) \theta^{i-1}\right)+O(1),
$$




$$
\left|\sigma^{i-1}\left(m_{i}\right)\right|-\varepsilon\left(m_{i}\right) \theta^{i-1}=O\left(i^{\alpha_{2}-1}\left|\theta_{2}\right|^{i}\right) \quad(\text { see Remarks } 1 \text { and } 2) .
$$

Hence $l^{k}|N-x|=O\left(\varphi_{k}(l)\right)$.

$F_{k, h}^{1}$ is bounded because $\left|\theta^{-l}\left(x_{i}+f(m)\right)^{k-q}(i-l)^{p-h} \theta^{i}\right|=O\left((n-i)^{k} \theta^{i-n}\right)$ and $\sum_{i=-\infty}^{n-2}(n-i)^{k} \theta^{i-n}$ does not depend on $n$. In the same way, $F_{k, h}^{2}$ is bounded.

Periodicity. Let $l^{\prime}=l+1$ and $x^{\prime}=\theta^{l^{\prime}}$. The representation of $x^{\prime}$ is connected with that of $x$ by $n\left(x^{\prime}\right)=n(x)+1$ and $m_{i}^{\prime}=m_{i-1}$ for $i \leq n+1$; so $S\left(x^{\prime}, i, p, q\right)=S(x, i-1, p, q)$ and $F_{k, h}^{1}(l+1)=F_{k, h}^{1}(l)$, and similarly for $F_{k, h}^{2}$.

Remark 3 . In the case where $s^{f}$ is the sum-of-digits in the $q$-ary expansion (see Section 1 ), one has $\theta=q$ and $\# \mathcal{A}=1$; so in Lemma $3, d^{\prime}=1$ and $\left(V_{n}^{k}\right)_{a}=p_{1 k a}(n) q^{n}$.

The representation of real numbers in Lemma $4\left(x=\sum_{i=-\infty}^{n} \varepsilon\left(m_{i}\right) \theta^{i-1}\right)$ coincides with their $q$-ary expansion. If $x$ is an integer, $m_{i}$ is empty for $i \leq 0$; then in the proof of Proposition 1, we can replace the condition $-\infty<i$ by $0 \leq i$. We obtain

$$
S_{k, \lambda}^{f}(x)=x \sum_{h=0}^{k} l^{h} F_{k, h}(l) \quad \text { for any integer } x \geq 1 \text { and } l=\log _{\theta} x .
$$

\section{Continuity}

Proposition 2. The functions $F_{k, h}$ are continuous on $\mathbb{R}$.

Proof. Let $\widetilde{S}:[1,+\infty[\rightarrow \mathbb{R}$ be the continuous piecewise affine function such that

$$
\widetilde{S}(n)=S_{k, \lambda}^{f}(n) \quad \text { for any } n \in \mathbb{N} .
$$

As $\widetilde{S}(x)=S_{k, \lambda}^{f}(x)+O\left(\varphi_{k}(l)\right)$ we can replace, in Proposition $1, S_{k, \lambda}^{f}(x)$ by $\widetilde{S}(x)$. Next we define, for $0 \leq h<k$, the functions

$$
\widetilde{S}_{k, h}(x)=\widetilde{S}(x)-x \sum_{h^{\prime}=h+1}^{k} l^{h^{\prime}} F_{k, h^{\prime}}(l) \quad\left(x \in \left[1,+\infty\left[, l=\log _{\theta} x\right) .\right.\right.
$$

If $h=k$, then $\widetilde{S}_{k, k}(x)$ is equal to $\widetilde{S}(x)$, which is continuous.

We want to establish a relation between $\widetilde{S}_{k, h}$ and $F_{k, h}$. We deduce from Proposition 1 that

$$
\widetilde{S}_{k, h}(x)=x l^{h} F_{k, h}(l)+O\left(x|l|^{h-1}\right),
$$

or, equivalently,

$$
F_{k, h}(l)=\theta^{-l} l^{-h} \widetilde{S}_{k, h}\left(\theta^{l}\right)+O\left(|l|^{-1}\right)
$$


hence

$$
F_{k, h}(l+n)=\theta^{-l-n}(l+n)^{-h} \widetilde{S}_{k, h}\left(\theta^{l+n}\right)+O\left(|l+n|^{-1}\right) \quad \text { for } n \in \mathbb{N} .
$$

But $F_{k, h}(l+n)=F_{k, h}(l)$. Moreover, given a compact set $K$ and $\varepsilon>0$ we have, for $n$ large enough, $|l+n|^{-1}<\varepsilon$ for any $l \in K$. In other words,

$F_{k, h}(l)=\lim _{n \rightarrow+\infty}\left(\theta^{-l-n}(l+n)^{-h} \widetilde{S}_{k, h}\left(\theta^{l+n}\right)\right) \quad$ (uniformly on compact sets).

By this relation and by (1), we obtain successively the continuity of $F_{k, k}$, $\widetilde{S}_{k, k-1}, F_{k, k-1}, \ldots, \widetilde{S}_{k, 0}, F_{k, 0}$.

5. The main result. We can specify the asymptotic expansion given in Proposition 1 , by computing $F_{k, h}(l)$ for the maximal $h$ such that $F_{k, h} \neq 0$. We will use the eigenvectors of $M . M$ is primitive, hence has a unique row-eigenvector $\xi$ defined by

$$
\xi M=\theta \xi \quad \text { and } \quad \xi\left(\begin{array}{c}
1 \\
\vdots \\
1
\end{array}\right)=1 .
$$

The vector $\varepsilon=\left(\varepsilon_{a}\right)_{a \in \mathcal{A}}$ is a column-eigenvector since, using Remarks 1 and 2 , we have

$$
\varepsilon=\lim _{n \rightarrow+\infty} \theta^{-n} M^{n}\left(\begin{array}{c}
1 \\
\vdots \\
1
\end{array}\right),
$$

hence $M \varepsilon=\theta \varepsilon$ and $\xi \varepsilon=1$. We define the constant

$$
\alpha=\theta^{-1} \sum_{a, m, b} \xi_{a} f(m) \varepsilon_{b} \quad\left(\text { sum over }(a, m, b) \in \mathcal{A} \times \mathcal{A}^{*} \times \mathcal{A} \text { with } m b \leq \sigma a\right) .
$$

In the case $\lambda=\alpha$, we will also use

$$
\beta=\theta^{-1} \xi\left(A^{(2)} \varepsilon+2 A^{(1)} v\right)
$$

where the vector $v$ is defined, modulo $\mathbb{R} \varepsilon$, by

$$
(\theta I-M) v=A^{(1)} \varepsilon .
$$

$\beta$ does not depend on the choice of $v$, because

$$
\theta^{-1} \xi A^{(1)} \varepsilon=\alpha-\lambda,
$$

which is zero in the case $\lambda=\alpha$. Such a $v$ exists: consider the hyperplane $(\theta I-M)\left(\mathbb{R}^{d}\right)$ and the hyperplane orthogonal to $\xi$, which contains the vector $A^{(1)} \varepsilon$ in the case $\lambda=\alpha$.

THEOREM. There exist continuous functions $F_{k, h}$ and $G_{k, h}$ with period 1 such that (as $x \rightarrow+\infty$ ) 


$$
\begin{gathered}
S_{k, 0}^{f}(x)=(\alpha l)^{k} x+x \sum_{h<k} l^{h} F_{k, h}(l)+O\left(\varphi_{k}(l)\right), \\
S_{k, \alpha}^{f}(x)= \begin{cases}\frac{k !}{(k / 2) !}\left(\frac{\beta l}{2}\right)^{k / 2} x+x \sum_{h<k / 2} l^{h} G_{k, h}(l)+O\left(\varphi_{k}(l)\right) & \text { if } k \text { is even, } \\
x \sum_{h<k / 2} l^{h} G_{k, h}(l)+O\left(\varphi_{k}(l)\right) & \text { if } k \text { is odd, }\end{cases}
\end{gathered}
$$

with $l=\log _{\theta} x$ and $\varphi_{k}(l)$ defined in Proposition 1 .

The next lemmas concern the polynomial of Lemma 3(ii). By (ii) and (iii) of that lemma, there exists a polynomial $P_{k}(X)=\sum_{p=0}^{k} b_{p}(k) X^{p}$ with $\mathbb{R}^{d}$ coefficients $b_{p}(k)$ such that

$$
V_{n}^{k}=\theta^{n} P_{k}(n)+o\left(\theta^{n}\right) .
$$

Writing now the formula of Lemma 1 modulo $o\left(\theta^{n}\right)$, and using the fact that two polynomials asymptotically equal are identical, we obtain

LEMMA 5.

$$
\theta P_{k}(n+1)=M P_{k}(n)+\sum_{h<k}\left(\begin{array}{l}
k \\
h
\end{array}\right) A^{(k-h)} P_{h}(n) .
$$

Next we compute the term of degree $k$ in $P_{k}(X)$.

LEMMA 6.

$$
b_{k}(k)=(\alpha-\lambda)^{k} \varepsilon
$$

Proof. This is true for $k=0$ (see Remark 2). Suppose

$$
b_{k-1}(k-1)=(\alpha-\lambda)^{k-1} \varepsilon .
$$

Identifying the terms of degree $k$ in the formula of Lemma 5 , we obtain

$$
\theta b_{k}(k)=M b_{k}(k) .
$$

Thus $b_{k}(k)$ is an eigenvector and there exists $t \in \mathbb{R}$ such that $b_{k}(k)=t \varepsilon$ ( $\theta$ being a simple eigenvalue by Perron-Frobenius).

Identifying the terms of degree $k-1$ in the formula of Lemma 5 , we obtain

$$
\theta k b_{k}(k)+\theta b_{k-1}(k)=M b_{k-1}(k)+k A^{(1)} b_{k-1}(k-1)
$$

and, multiplying on the left by $\xi$ and using $\xi \varepsilon=1$ and $\xi M=\theta \xi$,

$$
\theta k t+\theta \xi b_{k-1}(k)=\theta \xi b_{k-1}(k)+k \xi A^{(1)} b_{k-1}(k-1) .
$$

Then we can compute $t$, and from (1) and (2) we obtain the assertion. 
LEMma 7. If $\lambda=\alpha$, we have $d^{\circ}\left(P_{k}\right) \leq[k / 2]$ and

$$
\begin{aligned}
b_{[k / 2]}(k) & =\beta_{k} \varepsilon \quad \text { if } k \text { is even, } \\
(\theta I-M) b_{[k / 2]}(k) & =\beta_{k} A^{(1)} \varepsilon \quad \text { if } k \text { is odd, with } \beta_{k}=\frac{k !}{[k / 2] !}\left(\frac{\beta}{2}\right)^{[k / 2]} .
\end{aligned}
$$

Proof. For $k=0$, this formula is the same as in Lemma 6. Suppose $k \geq 1$ and the formula is true for $0,1, \ldots, k-1$. Then, by Lemma 5 , the degree of the polynomial $\theta P_{k}(n+1)-M P_{k}(n)$ is at most $k^{\prime}=[(k-1) / 2]$.

Let $p=d^{\circ}\left(P_{k}\right)$. If $p \geq k^{\prime}+2$ we obtain, identifying the terms of degree $p$ and $p-1$ in Lemma 5 ,

$$
\theta b_{p}(k)=M b_{p}(k) \quad \text { and } \quad \theta p b_{p}(k)+\theta b_{p-1}(k)=M b_{p-1}(k) .
$$

By the same computation as in Lemma 6 , we obtain $b_{p}(k)=0$, contrary to $p=d^{\circ}\left(P_{k}\right)$.

Hence $p \leq k^{\prime}+1$. Identifying the terms of degree $k^{\prime}+1$ and $k^{\prime}$ in Lemma 5, we obtain

$$
\begin{gathered}
\theta b_{k^{\prime}+1}(k)=M b_{k^{\prime}+1}(k) \\
\theta\left(k^{\prime}+1\right) b_{k^{\prime}+1}(k)+\theta b_{k^{\prime}}(k)-M b_{k^{\prime}}(k) \\
= \begin{cases}k A^{(1)} b_{k^{\prime}}(k-1) & \text { if } k \text { is odd } \\
k A^{(1)} b_{k^{\prime}}(k-1)+\frac{k(k-1)}{2} A^{(2)} b_{k^{\prime}}(k-2) & \text { if } k \text { is even. }\end{cases}
\end{gathered}
$$

By the same computation as in Lemma 6 , we obtain $b_{k^{\prime}+1}(k)=0$ if $k$ is odd (using (1), (3) and $b_{k^{\prime}}(k-1) \in \mathbb{R} \varepsilon$ by the induction hypothesis). Then by (3) we obtain the value of $(\theta I-M) b_{k^{\prime}}(k)$ and prove the assertion of the lemma for $k$.

If $k$ is even we again have $b_{k^{\prime}+1}(k)=t \varepsilon$ with $t \in \mathbb{R}$ and, multiplying (4) by $\xi$ on the left,

$$
\theta\left(k^{\prime}+1\right) t=k \xi A^{(1)} b_{k^{\prime}}(k-1)+\frac{k(k-1)}{2} \xi A^{(2)} b_{k^{\prime}}(k-2) .
$$

By the induction hypothesis, the vector $v=\left(1 / \beta_{k-1}\right) b_{k^{\prime}}(k-1)$ satisfies $(\theta I-M) v=A^{(1)} \varepsilon$, and the vector $b_{k^{\prime}}(k-2)$ is equal to $b_{(k-2) / 2}(k-2)=$ $\beta_{k-2} \varepsilon$. Using the definition of $\beta$ we obtain the assertion.

Proof of the Theorem. We compute $F_{k, k}(l)$, replacing $h$ by $k$ in the proof of Proposition 1. We obtain

$$
F_{k, k}^{1}(l)=\theta^{-l} \sum_{i, m, a} b_{k}(k, a) \theta^{i} \quad\left(\text { sum over }-\infty<i \leq n-2 \text { and } m a \leq m_{i+1}\right)
$$


and, by Lemma 6 ,

$$
F_{k, k}^{1}(l)=\theta^{-l}(\alpha-\lambda)^{k} \sum_{i=-\infty}^{n-2} \varepsilon\left(m_{i+1}\right) \theta^{i} .
$$

In the same way,

$$
F_{k, k}^{2}(l)=\theta^{-l}(\alpha-\lambda)^{k}\left(\left(\varepsilon\left(m_{n}\right)-\varepsilon(1)\right) \theta^{n-1}+\sum_{i=-\infty}^{n-2}(\theta-1) \varepsilon(1) \theta^{i}\right) .
$$

Hence $F_{k, k}(l)=(\alpha-\lambda)^{k}$ and we obtain the conclusion of the Theorem in the case $\lambda=0$.

In the case $\lambda=\alpha$, we first compute $F_{k, h}(l)$ for $h>[k / 2]$. This condition, together with $h \leq p \leq q \leq k$, implies $p>[q / 2]$; then $b_{p}(q, a)$ is zero by Lemma 7 , and $F_{k, h}(l)=0$. Next we compute $F_{k, k / 2}(l)$ for $k$ even. The condition $k / 2 \leq p \leq q \leq k$ implies $p=q / 2=k / 2$, else $p>q / 2$ and $b_{p}(q, a)=0$. The computation of $F_{k, k / 2}(l)$ is the same as that of $F_{k, k}(l)$ and leads to $F_{k, k / 2}(l)=\beta_{k}$.

Now we will check that the sum

$$
Z_{k, \alpha}^{f}(x)=\sum_{0<\nu<x}\left(s^{f}(\nu)-\alpha \log _{\theta} \nu\right)^{k}
$$

has the same equivalent as $S_{k, \alpha}^{f}(x)$ if $k$ is even. This sum is studied in [D90] and [GoL87]. then

Corollary. $Z_{k, \alpha}^{f}(x)=O\left(l^{[k / 2]} x\right)\left(\right.$ with $\left.l=\log _{\theta} x\right)$, and if $k$ is even,

$$
Z_{k, \alpha}^{f}(x)=\frac{k !}{(k / 2) !}\left(\frac{\beta l}{2}\right)^{k / 2} x+O\left(l^{k / 2-1} x\right) .
$$

Proof. It is sufficient to prove this for integer $x$. We have

$$
\begin{aligned}
Z_{k, \alpha}^{f}(x) & =\sum_{0<\nu<x}\left(\lambda_{\nu}+\mu_{\nu}\right)^{k} \quad \text { with } \lambda_{\nu}=s^{f}(\nu)-\alpha l \text { and } \mu_{\nu}=\alpha \log _{\theta}(x / \nu) \\
& =S_{k, \alpha}^{f}(x)+\sum_{0<\nu<x} \sum_{i=0}^{k-1}\left(\begin{array}{c}
k \\
i
\end{array}\right) \lambda_{\nu}^{i} \mu_{\nu}^{k-i} .
\end{aligned}
$$

Thus, it is sufficient to prove that for $i \leq k-1$ and $j \leq k, \sum_{0<\nu<x} \lambda_{\nu}^{i} \mu_{\nu}^{j}=$ $O\left(l^{[(k-1) / 2]} x\right)$. We remark that

$$
\sum_{0<\nu<x} \lambda_{\nu}^{i} \mu_{\nu}^{j}=\sum_{0<\nu<x}\left(\mu_{\nu}^{j}-\mu_{\nu+1}^{j}\right) \sum_{\nu^{\prime}=1}^{\nu} \lambda_{\nu^{\prime}}^{i} .
$$

We deduce from the theorem that $\sum_{\nu^{\prime}=1}^{\nu} \lambda_{\nu^{\prime}}^{i}=O\left(\nu l^{[i / 2]} \mu_{\nu}^{i}\right)$, and from the 
mean value theorem

$$
\mu_{\nu}^{j}-\mu_{\nu+1}^{j}=O\left(\mu_{\nu}^{j-1} / \nu\right)
$$

Hence

$$
\sum_{0<\nu<x} \lambda_{\nu}^{i} \mu_{\nu}^{j}=O\left(l^{[i / 2]} \sum_{0<\nu<x} \mu_{\nu}^{i+j-1}\right)
$$

In the case $i=0$ this estimate becomes

$$
\sum_{0<\nu<x} \mu_{\nu}^{j}=O\left(\sum_{0<\nu<x} \mu_{\nu}^{j-1}\right)
$$

and by induction $\sum_{0<\nu<x} \mu_{\nu}^{j}=O(x)$. Then we deduce from (1) that

$$
\sum_{0<\nu<x} \lambda_{\nu}^{i} \mu_{\nu}^{j}=O\left(l^{[(k-1) / 2]} x\right) .
$$

R e m a r k. A more precise computation should give the existence of functions $H_{k, h}$ such that

$$
Z_{k, \alpha}^{f}(x)=\frac{k !}{(k / 2) !}\left(\frac{\beta l}{2}\right)^{k / 2} x+x \sum_{h<k / 2} l^{h} H_{k, h}(l)+O\left(\varphi_{k}(l)\right)
$$

for $k$ even, and the same without the first term of the r.h.s. if $k$ is odd.

The functions $H_{k, h}$ are related to the functions $G_{k, h}$ of the Theorem (and $G_{k, k / 2}=\frac{k !}{(k / 2) !}\left(\frac{\beta}{2}\right)^{k / 2}$ if $k$ even):

$$
\begin{aligned}
H_{k, h}(l)=G_{k, h}(l)-\sum\left(\begin{array}{c}
k \\
i
\end{array}\right) & \left(\begin{array}{c}
k-i \\
k-i^{\prime}
\end{array}\right)\left(\begin{array}{l}
j \\
h
\end{array}\right) \frac{i(-1)^{i} \alpha^{i^{\prime}}}{\theta^{l} \log \theta} \\
& \times \int_{0}^{\theta^{l}}\left(\log _{\theta} \nu-l\right)^{i^{\prime}+j-h-1} G_{k-i^{\prime}, j}\left(\log _{\theta} \nu\right) d \nu
\end{aligned}
$$

(sum over $h \leq j \leq[(k-1) / 2]$ and $1 \leq i \leq i^{\prime} \leq k-2 j$ ).

Of course $H_{k, h}$ is periodic and continuous, and differentiable iff $G_{k, h}$ is.

\section{Nondifferentiability of $F_{k, h}$ and $G_{k, h}$}

Proposition 3. If $\alpha \neq 0$ and $\alpha \neq f(\omega)$, then the functions $F_{k, h}$ of the Theorem are nowhere differentiable for $h<k$. If $\beta \neq 0$ and $\alpha \neq f(\omega)$, then $G_{k, h}$ are nowhere differentiable for $h<k / 2$.

Proof. For fixed $k$ and $h<k$, we define a mapping $(x, l) \rightarrow \phi_{x}(l)$ from $\mathbb{R}_{+}^{*} \times \mathbb{R}$ to $\mathbb{R}$ such that, in the case $x=\theta^{l}, \phi_{x}(l)$ should be equal to $x F_{k, h}(l)$ (with $F_{k, h}(l)$ defined in the proof of Proposition 1).

We set

$$
\phi_{x}(l)=\sum_{i=-\infty}^{n-1} \phi_{x, i}(l), \quad \phi_{x, i}(l)=\phi_{x, i}^{1}(l)+\phi_{x, i}^{2}(l),
$$


$\phi_{x, i}^{1}(l)$

$$
=\left\{\begin{array}{l}
\sum_{h \leq p \leq q \leq k} \sum_{m a \leq m_{i+1}}\left(\begin{array}{l}
k \\
q
\end{array}\right)\left(x_{i, m}-\lambda(l-i)\right)^{k-q}\left(\begin{array}{l}
p \\
h
\end{array}\right) b_{p}(q, a)(i-l)^{p-h} \theta^{i} \\
\quad \text { if } i \leq n-2 \\
0 \quad \text { if } i=n-1
\end{array}\right.
$$

(where $x_{i, m}=f\left(m_{n}\right)+\ldots+f\left(m_{i+2}\right)+f(m)$ depends on the representation $\left(m_{i}\right)_{-\infty<i \leq n}$ of $x$, but not on $\left.l\right)$.

$\phi_{x, i}^{2}(l)$ is defined in the same way as $\phi_{x, i}^{1}(l)$, with $x_{i, m}$ replaced by $f(m)$, and with the condition $m a \leq m_{i+1}$ replaced by $1 \leq m<m a \leq \sigma(1)$, or $1 \leq m<m a \leq m_{n}$ if $i=n-1$.

$\phi_{x, i}^{1}(l)$ is a polynomial in $l$ whose coefficients have absolute value less than $C(|i|+1)^{k} \theta^{i}$, with $C$ a constant independent of $i$ and $l$. As the series $\sum_{i=-\infty}^{n-1}(|i|+1)^{k} \theta^{i}$ converges, we deduce that $\phi_{x}(l)$ is also a polynomial in $l$.

Suppose $F_{k, h}$ is differentiable at the point $l$; let $x=\theta^{l}$. Then $x$ has a representation $m_{n} m_{n-1} \ldots=\left(m_{i}\right)_{-\infty<i \leq n}$ by Lemma 4 .

For any $j \leq n$, we define two real numbers $u_{j}$ and $v_{j}: u_{j}$ has representation $m_{n} m_{n-1} \ldots m_{j} \omega^{\mathbb{N}}$, and $v_{j}$ has representation $m_{n} m_{n-1} \ldots m_{j} \omega^{\nu-1} a \omega^{\mathbb{N}}$.

Fix now $\nu$ such that $\left|\sigma^{\nu}(b)\right| \geq 2$ for any $b \in \mathcal{A}$, and set $a=1 \cdot m_{n} \cdot \ldots$ $\ldots \cdot m_{j} \cdot \omega^{\nu}$; then $m_{n} \ldots m_{j} \omega^{\nu-1} a \omega^{\mathbb{N}}$ is 1-recognizable. We have

$$
v_{j}-u_{j}=\varepsilon(a) \theta^{j-\nu-1} \text {. }
$$

Let

$$
\Delta_{j}=\frac{v_{j} F_{k, h}\left(l_{j}^{\prime}\right)-u_{j} F_{k, h}\left(l_{j}\right)}{v_{j}-u_{j}} \quad\left(l_{j}^{\prime}=\log _{\theta} v_{j} \text { and } l_{j}=\log _{\theta} u_{j}\right) .
$$

This is the rate of variation of the function $t \rightarrow t F_{k, h}\left(\log _{\theta} t\right)$ between the points $u_{j}$ and $v_{j}$. As $j$ tends to $-\infty, u_{j}-x=O\left(\left|v_{j}-u_{j}\right|\right)$; thus $\Delta_{j}$ tends to the derivative of this function at the point $x$.

We will deduce that the rate of variation

$$
\Delta_{j}^{\prime}=\frac{\phi_{v_{j}}\left(l_{j}\right)-\phi_{u_{j}}\left(l_{j}\right)}{v_{j}-u_{j}}
$$

also has a limit. By the mean value theorem, there exists a real number $l_{j}^{\prime \prime}$ between $l_{j}$ and $l_{j}^{\prime}$ such that

$$
\Delta_{j}-\Delta_{j}^{\prime}=\frac{\left(l_{j}^{\prime}-l_{j}\right) \phi_{v_{j}}^{\prime}\left(l_{j}^{\prime \prime}\right)}{v_{j}-u_{j}}
$$

(where $\phi_{v_{j}}^{\prime}$ is the derivative of the function $t \rightarrow \phi_{v_{j}}(t)$ ). We have

$$
\phi_{v_{j}}^{\prime}\left(l_{j}^{\prime \prime}\right)=\phi_{x}^{\prime}\left(l_{j}^{\prime \prime}\right)+O\left(\left|l_{j}^{\prime \prime}-j\right|^{k} \theta^{j}\right)
$$


(since the representations of $v_{j}$ and $x$ coincide between the indices $j$ and $n$ ). As $j$ tends to $-\infty, \phi_{x}^{\prime}\left(l_{j}^{\prime \prime}\right)$ tends to $\phi_{x}^{\prime}(l)$, since $\phi_{x}^{\prime}$ is a polynomial. Thus $\phi_{v_{j}}^{\prime}\left(l_{j}^{\prime \prime}\right) \rightarrow \phi_{x}^{\prime}(l), \Delta_{j}-\Delta_{j}^{\prime} \rightarrow(x \ln \theta)^{-1} \phi_{x}^{\prime}(l)$ and

$$
\Delta_{j}^{\prime} \rightarrow L=F_{k, h}(l)+(\ln \theta)^{-1} F_{k, h}^{\prime}(l)-(x \ln \theta)^{-1} \phi_{x}^{\prime}(l) .
$$

Now

$$
\begin{array}{r}
\Delta_{j}^{\prime}=\varepsilon(a)^{-1} \sum_{h \leq p \leq q \leq k}\left(\begin{array}{l}
k \\
q
\end{array}\right)\left(x_{j, \omega}+(\nu-1) f(\omega)-\lambda\left(l_{j}-j+\nu+1\right)\right)^{k-q} \\
\times\left(\begin{array}{l}
p \\
h
\end{array}\right) b_{p}(q, a)\left(j-\nu-1-l_{j}\right)^{p-h} .
\end{array}
$$

Fixing $j$, we consider $\Delta_{j}^{\prime}$ as a polynomial in $\nu$; its degree is at most $k-h$. We compute the coefficient $c_{k-h}$ of the term of degree $k-h$; it is obtained for $p=q$; using Lemma 6 and the binomial formula we obtain

$$
\left.c_{k-h}=\left(\begin{array}{l}
k \\
h
\end{array}\right)(f(\omega)-\alpha)^{k-h}(\alpha-\lambda)^{h} \quad \text { (independent of } j\right) .
$$

But this coefficient is also equal to $(1 /(k-h) !) \Delta^{k-h}\left(\Delta_{j}^{\prime}\right)$, where $\Delta$ is the operator which associates with every polynomial $P(X)$ the polynomial $P(X+1)-P(X)$. As $\lim _{j \rightarrow-\infty} \Delta^{k-h}\left(\Delta_{j}^{\prime}\right)=\Delta^{k-h}(L)=0$, we obtain $c_{k-h}=0$.

So if the function $F_{k, h}$ of Proposition 1 is differentiable, we have necessarily $\alpha=f(\omega)$ or $\lambda=\alpha$.

In the case $\alpha=f(\omega)$ a counterexample is given in [DT91].

In the case $\lambda=\alpha$, we must prove that $F_{k, h}$ is not differentiable for $h<k / 2$ except in the cases $\alpha=f(\omega)$ or $\beta=0$. We have seen (Section 5) that $b_{p}(q, a)$ is zero if $p>q / 2$; hence $\Delta_{j}^{\prime}$ is a polynomial in $\nu$ of degree at most $k-2 h$, because $k-q+p-h \leq k-2 p+p-h \leq k-2 h$; the term of degree $k-2 h$ is obtained for $p=h$ and $q=2 h$. As $h<k / 2, k-2 h$ is positive hence $\Delta^{k-2 h}(L)=0$.

We deduce that

$$
\begin{gathered}
0=\lim _{j \rightarrow-\infty} \Delta^{k-2 h}\left(\Delta_{j}^{\prime}\right)=(k-2 h) ! \varepsilon(a)^{-1}\left(\begin{array}{c}
k \\
2 h
\end{array}\right)(f(\omega)-\alpha)^{k-2 h} b_{h}(2 h, a), \\
b_{h}(2 h, a)=\frac{(2 h) !}{h !}\left(\frac{\beta}{2}\right)^{h} \varepsilon(a),
\end{gathered}
$$

hence $\alpha=f(\omega)$ or $\beta=0$.

7. Application to the sequence $(n \omega)_{n \geq 1}$ for some quadratic $\omega$. Consider the sequence $\varepsilon=\left(\varepsilon_{n}\right)_{n \geq 1}$ defined by

$$
\varepsilon_{n}= \begin{cases}1 & \text { if } \operatorname{frac}(n \omega / 2)<1 / 2 \\ -1 & \text { otherwise }\end{cases}
$$


where $\omega$ is a quadratic number such that

$$
\omega=\frac{1}{a_{1}+\frac{1}{a_{2}+\omega}} \quad \text { with } a_{1} \in \mathbb{N}^{*}, a_{2}=2 \nu, \nu \in \mathbb{N}^{*} .
$$

Godrèche, Luck and Vallet ([GoL87]) asked about the asymptotic expansion of

$$
\frac{1}{N} \sum_{n=1}^{N}\left(s(n)-\frac{a_{1}}{2} \log _{\theta} n\right)^{2}
$$

where $s(n)=\sum_{i=1}^{n} \varepsilon_{i}$ and $\theta=a_{1}\left(a_{2}+\omega\right)+1$.

We obtain (Section 5)

$$
\frac{1}{N} \sum_{n=1}^{N}\left(s(n)-\frac{a_{1}}{2} \log _{\theta} n\right)^{2}=\beta \log _{\theta} N+H_{2,0}\left(\log _{\theta} N\right)+o(1)
$$

with

$$
\beta=\frac{\left(\theta-a_{1} \nu-1\right)\left(a_{1}^{2} \nu+3 a_{1}+2 \nu\right)}{12 a_{1} \nu\left(a_{1} \nu+2\right)} .
$$

Indeed, the sequence $\varepsilon$ may be obtained from the substitution $\sigma$ on the alphabet $\mathcal{A}=\{1,2,3\}$ defined by

$$
\begin{gathered}
\sigma(1)=m^{\nu} 1 \quad\left(\text { where } m=1^{a_{1}} 23^{a_{1}-1}\right), \\
\sigma(2)=m^{\nu+1} 3, \quad \sigma(3)=m^{\nu} 3
\end{gathered}
$$

and the output function

$$
f(1)=1, \quad f(2)=f(3)=-1
$$

(i.e. $\varepsilon_{i}=f\left(u_{i}\right)$ where $u_{1}=1$ and $\left(u_{i}\right)_{i \geq 1}$ is the fixed point of $\sigma$ ).

This substitution is the same as the one of [GoL87], Section 4 ( $a_{2}$ even), upon replacing their letters $a$ and $c$ by the letter $1, b$ by 2 and $d$ by 3 .

The matrix of $\sigma$ is

$$
M=\left(\begin{array}{ccc}
a_{1} \nu+1 & \nu & \left(a_{1}-1\right) \nu \\
a_{1}(\nu+1) & \nu+1 & a_{1}(\nu+1)-\nu \\
a_{1} \nu & \nu & \left(a_{1}-1\right) \nu+1
\end{array}\right)
$$

the eigenvectors defined in Section 5 are here

$$
\xi=\left(\frac{1}{2}, \frac{\theta-1}{2 a_{1}}-\nu, \frac{1}{2}+\nu-\frac{\theta-1}{2 a_{1}}\right) \quad \text { and } \quad \varepsilon=\frac{\theta}{\theta+1}\left(\begin{array}{c}
1 \\
1+\frac{1}{\nu}-\frac{1}{\theta \nu} \\
1
\end{array}\right) .
$$

The condition $f(\sigma(m))=f(m)$ (see Section 1) is satisfied. An easy calculation gives, for $\alpha$ and $\beta$ defined in Section $5, \alpha=a_{1} / 2$ and $\beta$ as indicated above. 


\section{Sum-of-digits function in the case of finite Parry expansion.} Now we compute $\alpha$ and $\beta$ in the case of "normal numeration" associated with linear finite recurrence expansion with canonical initial values (cf. [B89] and [GTi91]; see also [Fro] and [Sh88]).

Let $\left(u_{i}\right)_{i \geq 1}$ be a strictly increasing sequence of positive integers with $u_{1}=1$; the normal representation of the integer $N \geq 1$ with respect to $\left(u_{i}\right)_{i \geq 1}$ is the finite sequence of $n$ integers $\varepsilon_{1}, \varepsilon_{2}, \ldots, \varepsilon_{n}$ uniquely determined by the "greedy algorithm":

$$
u_{n} \leq N<u_{n+1}, \quad \varepsilon_{n}=\left[N / u_{n}\right], \quad r_{n}=N-\varepsilon_{n} u_{n},
$$

and, for $2 \leq i \leq n$,

$$
\varepsilon_{i-1}=\left[r_{i} / u_{i-1}\right], \quad r_{i-1}=r_{i}-\varepsilon_{i-1} u_{i-1} .
$$

If $\left(\varepsilon_{i}^{\prime}\right)_{i=1, \ldots, n}$ are integers such that $N=\sum_{i=1}^{n} \varepsilon_{i}^{\prime} u_{i}$, then the sequence $\left(\varepsilon_{i}^{\prime}\right)_{i=1, \ldots, n}$ is the normal representation of $N$ if and only if

$$
\forall j=1, \ldots, n \quad \sum_{i=1}^{j} \varepsilon_{i}^{\prime} u_{i}<u_{j+1} \quad \text { (cf. [Fr]). }
$$

Now let $d \geq 1$ be an integer and $a_{1}, a_{2}, \ldots, a_{d}$ non-negative integers with $a_{d} \geq 1$ satisfying the "Parry condition": if $d \geq 2$, then

$$
\forall j=2, \ldots, d \quad a_{j} \ldots a_{d} 0^{j-1}<_{l} a_{1} a_{2} \ldots a_{d}
$$

$\left(<_{l}\right.$ being the lexicographic order), and if $d=1$, then $a_{1} \geq 2$.

Let $\left(u_{i}\right)_{i \geq 1}$ be such that $u_{1}=1$ and

$$
u_{i}= \begin{cases}a_{1} u_{i-1}+a_{2} u_{i-2}+\ldots+a_{i-1} u_{1}+1 & (2 \leq i \leq d) \\ a_{1} u_{i-1}+a_{2} u_{i-2}+\ldots+a_{d} u_{i-d} & (i>d)\end{cases}
$$

Then $\left(u_{i}\right)_{i \geq 1}$ is strictly increasing (because $a_{1}, a_{d} \geq 1$ ).

Lemma. Define $\mathcal{A}=\{1,2, \ldots, d\}$ and let $\sigma$ be the substitution over $\mathcal{A}$ given by

$$
\sigma(j)= \begin{cases}1^{a_{j}}(j+1) & \text { if } 1 \leq j \leq d-1 \\ 1^{a_{d}} & \text { if } j=d\end{cases}
$$

(i) For any $i \geq 1, u_{i}=\left|\sigma^{i-1}(1)\right|$.

(ii) If $N=\sum_{i=1}^{n}\left|\sigma^{i-1}\left(m_{i}\right)\right|$ is the admissible representation of $N$ relative to the substitution $\sigma$, then the sequence $\varepsilon_{i}=\left|m_{i}\right|(i=1, \ldots, n)$ is the normal representation of $N$ with respect to $\left(u_{i}\right)_{i \geq 1}$.

P r o of. (i) Immediate using the definition of $\sigma$.

(ii) Using [DT89], Lemma 1.1, we have for $0 \leq j \leq n$

$$
\sum_{i=1}^{j}\left|\sigma^{i-1}\left(m_{i}\right)\right|<\left|\sigma^{j}\left(k_{j}\right)\right| \quad \text { for some } k_{j} \in \mathcal{A} \text {. }
$$


Thus, it remains to prove that for any $k \in \mathcal{A}$ and $j \in \mathbb{N}$,

$$
\left|\sigma^{j}(k)\right| \leq\left|\sigma^{j}(1)\right|
$$

We have

$$
\sigma^{j}(k)= \begin{cases}\left(\sigma^{j-1}(1)\right)^{a_{k}}\left(\sigma^{j-2}(1)\right)^{a_{k+1}} \ldots 1^{a_{k+j-1}}(k+j), & 0 \leq j \leq d-k, \\ \left(\sigma^{j-1}(1)\right)^{a_{k}}\left(\sigma^{j-2}(1)\right)^{a_{k+1}} \ldots\left(\sigma^{j-d+k-1}(1)\right)^{a_{d}}, & j>d-k .\end{cases}
$$

Now we will use the fact that for the admissible representation, a lexicographic inequality between two representations implies an ordinary inequality between the represented numbers.

If $k \geq 2$, then by the Parry condition in the first case

$$
\left|\sigma^{j-1}\left(1^{a_{k}}\right)\right|+\left|\sigma^{j-2}\left(1^{a_{k+1}}\right)\right|+\ldots+\left|1^{a_{k+j-1}}\right|
$$

is the admissible representation of $\left|\sigma^{j}(k)\right|-1$, and in the second case

$$
\left|\sigma^{j-1}\left(1^{a_{k}}\right)\right|+\left|\sigma^{j-2}\left(1^{a_{k+1}}\right)\right|+\ldots+\left|\sigma^{j-d+k-1}\left(1^{a_{d}}\right)\right|
$$

is the admissible representation of $\left|\sigma^{j}(k)\right|$. Moreover, these representations are $<_{l} 1 \omega^{j}$ and the proof is complete.

The matrix of $\sigma$ and the eigenvectors satisfying the conditions of Section 5 are

$$
M=\left(\begin{array}{ccccc}
a_{1} & 1 & 0 & \ldots & 0 \\
a_{2} & 0 & 1 & \ldots & 0 \\
\ldots & \ldots & \ldots & \ldots & . \\
a_{d} & 0 & 0 & \ldots & 0
\end{array}\right), \quad \xi=\frac{\theta-1}{\theta^{d}-1}\left(\theta^{d-1}, \ldots, \theta, 1\right)
$$

and

$$
\varepsilon=\frac{\theta^{d}-1}{(\theta-1) \theta P^{\prime}(\theta)}\left(\begin{array}{c}
\theta \\
\theta^{2}-a_{1} \theta \\
\vdots \\
\theta^{d}-a_{1} \theta^{d-1}-\ldots-a_{d-1} \theta
\end{array}\right)
$$

where $\theta$ is the root of $P(X)=X^{d}-a_{1} X^{d-1}-\ldots-a_{d}$ with maximum modulus.

We compute the constants $\alpha$ and $\beta$ of Section 5 ; the output function is here $f(m)=|m|$ and we check the vector $v$ with first coordinate 0 ; we obtain

$$
\begin{gathered}
\alpha=\frac{Q(\theta)}{\theta P^{\prime}(\theta)} \quad \text { with } Q(X)=a_{1}^{\prime} X^{d-1}+\ldots+a_{d}^{\prime} \text { and } \\
a_{i}^{\prime}=a_{i}\left(\frac{1}{2}\left(a_{i}-1\right)+\sum_{j<i} a_{j}\right), \\
\beta=\frac{R(\theta)}{\theta P^{\prime}(\theta)}-\alpha^{2} \quad \text { with } R(X)=a_{1}^{\prime \prime} X^{d-1}+\ldots+a_{d}^{\prime \prime}
\end{gathered}
$$


and

$$
\begin{gathered}
a_{i}^{\prime \prime}=a_{i}\left(\frac{1}{3}\left(a_{i}-1\right)\left(a_{i}-\frac{1}{2}\right)-\left(a_{i}-1-2 \alpha\right) A_{i}+\sum_{j<i}\left(a_{j}^{2}-2\left(a_{j}-\alpha\right) A_{j}\right)\right) \\
A_{i}=\sum_{k=i}^{d}\left(a_{k}-\alpha\right) .
\end{gathered}
$$

For instance, in the case of the ordinary numeration system $d=1$, $\sigma(1)=1^{q}$ and $\alpha=(q-1) / 2, \beta=\left(q^{2}-1\right) / 12$.

In the case of the Fibonacci expansion $d=2, \sigma(1)=12$ and $\sigma(2)=1$, $a_{1}=a_{2}=1, \alpha=(5-\sqrt{5}) / 10$ and $\beta=1 /(5 \sqrt{5})$.

Remark ([Fa92]). If $\left(a_{i}\right)_{i \geq 1}$ is an eventually periodic sequence $a_{1} \ldots a_{m}\left(a_{m+1} \ldots a_{m+l}\right)^{\infty}(m \geq 0, l \geq 1)$ satisfying the Parry condition

$$
\forall j \geq 2 \quad a_{j} a_{j+1} \ldots<_{l} a_{1} a_{2} \ldots
$$

and $\left(u_{i}\right)_{i \geq 1}$ the sequence $u_{1}=1, u_{i}=a_{1} u_{i-1}+a_{2} u_{i-2}+\ldots+a_{i-1} u_{1}+1$ $(i \geq 2)$, then the numeration associated with the substitution $\sigma$ on the finite alphabet $\mathcal{A}=\{1,2, \ldots, m+l\}$ given by

$$
\sigma(j)= \begin{cases}1^{a_{j}}(j+1) & (j=1,2, \ldots, m+l-1), \\ 1^{a_{m+l}}(m+1) & (j=m+l)\end{cases}
$$

is the same as the normal representation of integers with respect to $\left(u_{i}\right)_{i \geq 1}$ (same proof). We leave the computation of $\alpha$ and $\beta$ in this case to the reader.

\section{References}

[B89] A. Bertrand-Mathis, Comment écrire les nombres entiers dans une base qui n'est pas entière, Acta Math. Hungar. 54 (3-4) (1989), 237-241.

[C86] J. Coquet, Power sums of digital sums, J. Number Theory 22 (2) (1986), 161-176.

[CV86] J. Coquet and P. van den Bosch, A summation formula involving Fibonacci digits, ibid. 139-146.

[De75] H. Delange, Sur la fonction sommatoire de la fonction "somme des chiffres", Enseign. Math. (2) 21 (1) (1975), 31-47.

[D90] J. M. Dumont, Summation formulae for substitutions on a finite alphabet, in: Number Theory and Physics (Les Houches, 1989), Springer Proc. Phys. 47, Springer, Berlin 1990, 185-194.

[DT89] J. M. Dumont et A. Thomas, Systèmes de numération et fonctions fractales relatifs aux substitutions, Theoret. Comput. Sci. 65 (2) (1989), 153-169.

[DT91] - - - Digital sum problems and substitutions on a finite alphabet, J. Number Theory 39 (3) (1991), 351-366.

[Fa92] S. Fabre, Substitutions et indépendance des systèmes de numération, thèse, Faculté des sciences de Marseille-Luminy, 1992. 
[FG] P. Flajolet, P. Grabner, P. Kirschenhofer, H. Prodinger and R. Tichy, Mellin transforms and asymptotics: digital sums, Theoret. Comput. Sci., to appear.

[Fr] A. S. Fraenkel, Systems of numeration, Amer. Math. Monthly 92 (2) (1985), 105-114.

[Fro] C. Frougny, Representations of numbers and finite automata, Math. Systems Theory 25 (1) (1992), 37-60.

[GoL87] C. Godrèche, J. M. Luck and F. Vallet, Quasiperiodicity and types of order: a study in one dimension, J. Phys. A 20 (13) (1987), 4483-4499.

[GTi91] P. Grabner and R. Tichy, $\alpha$-expansions, linear recurrences, and the sum-ofdigits function, Manuscripta Math. 70 (3) (1991), 311-324.

[K90] P. Kirschenhofer, On the variance of the sum-of-digits function, in: Number Theory and Analysis, Lecture Notes in Math. 1452, Springer, Berlin 1990, 112116.

[L] J. M. Luck, Private communication.

[MM] J. L. Mauclaire and L. Murata, An explicit formula for the average of some q-additive functions, in: Prospects of Mathematical Science (Tokyo, 1986), World Sci., Singapore 1988, 141-156.

[Sh88] J. Shallit, A generalisation of automatic sequences, Theoret. Comput. Sci. 61 (1) (1988), 1-16.

Jean Marie Dumont

Alain Thomas

LABORATOIRE

UFR-MIM

DE MATHÉMATIQUES DISCRÈTES

FACULTÉ DES SCIENCES DE ST. CHARLES

UNITÉ PROPRE DE RECHERCHE 9016, CASE 930

163, AVENUE DE LUMINY

UNIVERSITÉ DE PROVENCE, CASE F

F-13288 MARSEILLE CEDEX 9

3, PLACE VICTOR HUGO

FRANCE

F-13331 MARSEILLE CEDEX 3

FRANCE

Received on 31.3.1992

and in revised form on 7.7.1992 